\title{
Triggers for Aggressive Interventions in Subarachnoid Hemorrhage
}

\author{
Nino Stocchetti - The participants in the International Multi-disciplinary Consensus \\ Conference on the Critical Care Management of Subarachnoid Hemorrhage
}

Published online: 12 July 2011

(C) Springer Science+Business Media, LLC 2011

\begin{abstract}
Ischemia is a common cause of secondary neuronal injury after aneurysmal subarachnoid hemorrhage. An electronic literature search was conducted to identify clinical signs and laboratory data that could serve as predictors for delayed cerebral ischemia and define triggers for additional diagnostic testing or more aggressive intervention. Fifteen articles describing original research that included some discussion of triggers were identified and reviewed. Quality of evidence was considered very low to moderate for included studies. Using data from these studies and expert opinion, a variety of clinical signs and monitoring data were identified as potentially useful triggers for additional tests or aggressive treatments. These data were used to develop a sequence that might be employed in the clinical management of subarachnoid hemorrhage to determine which patients need additional attention, testing, or interventions to reduce/prevent ischemia caused by vasospasm.
\end{abstract}

Keywords Early ischemia - Predictor - Risk factors . Vasospasm

The participants in the International Multi-disciplinary Consensus Conference: Michael N. Diringer, Thomas P. Bleck, Nicolas Bruder, E. Sander Connolly, Jr., Giuseppe Citerio, Daryl Gress, Daniel Hanggi, J. Claude Hemphill, III, MAS, Brian Hoh, Giuseppe Lanzino, Peter Le Roux, David Menon, Alejandro Rabinstein, Erich Schmutzhard, Lori Shutter, Nino Stocchetti, Jose Suarez, Miriam Treggiari, MY Tseng, Mervyn Vergouwen, Paul Vespa, Stephan Wolf, Gregory J. Zipfel.

N. Stocchetti ( $\square)$

Neuroscience ICU, Milan University, Fondazione IRCCS Cà Granda-Ospedale Policlinico, Via F. Sforza 35, Milan, Italy e-mail: stocchet@policlinico.mi.it

\section{Introduction}

Delayed cerebral ischemia (DCI) occurs frequently during the acute phase after subarachnoid hemorrhage (SAH) [1] and is linked to worse outcome, including increased mortality or disability $[2,3]$. Ischemia usually develops over time and, as such, might be amenable to treatment, provided early recognition and effective therapies are available.

Several mechanisms can be responsible for new ischemic deficits after SAH, including vasospasm and spreading depression. Progressive narrowing of an arterial lumen increases vascular resistance and leads to a decrease in blood flow if other hemodynamic factors are unchanged. When compensatory mechanisms, such as increased oxygen extraction, are exhausted, reduced blood flow translates into reduced oxygen delivery. When critical levels are reached, cellular metabolic integrity is compromised and clinical ischemia can occur.

Some experts have advocated for prophylactic treatment in patients with SAH to reduce ischemia risks [4]. Unfortunately, the rate of side-effects linked to therapies, such as induced arterial hypertension or metabolic suppression by barbiturates, is significant. Any potential benefits, therefore, must be weighed against potential undesirable complications.

A practical alternative to the use of prophylactic treatments is early diagnosis and rapid intervention when signs of DCI are identified. Early diagnosis is facilitated by recognizing signs and symptoms that may alert the treating team to imminent development of DCI. These signals or triggers could be used to prompt further diagnostic procedures and/or therapeutic interventions. This paper was designed to review available evidence regarding triggers and to propose a possible sequence, both diagnostic and therapeutic, for how to use 
these triggers in the clinical management of $\mathrm{SAH}$ to reduce secondary neuronal injury from ischemia.

\section{Methods}

An electronic PubMed literature was performed to identify articles describing triggers for ischemic changes in patients with SAH published through September 2010. An initial search was conducted using the key word "subarachnoid hemorrhage" plus "ischemic deficit" or "vasospasm." Candidate articles were limited to those that described clinical trials in adults and were written in English, French, Spanish, or Italian. A subsequent selection was made from those papers by using the key word "trigger" plus "subarachnoid" and/or "hemorrhage." Among identified articles, selections were made for appropriate articles to include by reviewing titles and abstracts.

Data were summarized and used to help develop a tiered system for utilizing trigger information in the clinical management of SAH. This sequence incorporated data from the literature, published guidelines, and the clinical expertise of the authors. Both the evidentiary table developed describing reviewed data and the levels of alarm sequence were sent to two external reviewers who are experts in the management of SAH patients for remarks and comments. The final version, reported here, incorporates their remarks.

\section{Summary of the Literature}

The initial search yielded 42 papers from the terms "subarachnoid hemorrhage" plus "ischemic deficit" and 3,511 from the terms "subarachnoid hemorrhage" with "vasospasm." Among these papers, 193 were identified using the study design and language restrictions. Among those, 41 papers were identified when including the "trigger" search. After reviewing the titles, 39 potential articles were identified, with a final total of 15 selected after reviewing abstracts. Data from these papers are summarized in Table 1, [1, 5-18].

In addition, a guideline was identified [19] to use to help determine appropriate clinical application of trigger information. This guideline, endorsed by the American Heart Association (AHA) and American Academy of Neurology, was an update of recommendations for managing aneurysmal SAH published by the AHA Stroke Council in 1994. This update was based on clinical trial data published through November 2006.

\section{Triggers for DCI in SAH}

None of the identified papers specifically addressed the topic of predictive triggers for DCI in patients with
SAH. Among the 15 papers reviewed, quality of evidence was moderate in 5 and low to very low in the remainder.

While the occurrence of neurological deficits after SAH could depend on several factors, for instance from spreading depolarization [20, 21], the authors decided to focus on ischemia related to arterial vasospasm when seeking triggers as this was most frequently reported in the selected papers. Several potential triggers were identified after determining which factors most consistently appeared to be linked with flow reduction, inadequate oxygen delivery, or clinical manifestations of ischemia (Table 2).

\section{Applying Trigger Identification to Clinical Management of SAH}

Trigger identification suggested a possible sequence of actions for clinical management. For example, Qureshi et al. proposed an index of vasospasm risk by utilizing data for clot thickness, neurologic presentation, site of aneurysm, and transcranial Doppler velocities [5]. Both clinical signs and monitoring data were used to develop a reaction sequence to potential triggers (Fig. 1).

The recommended sequence for reaction to triggers divided signs into Alarm level categories. Patients with an increased baseline risk were defined as Alarm level 1 patients, with a recommendation to observe more closely. Clinical deterioration, i.e., unexplained blood pressure rise and/or the appearance of a new deficit, raised the Alarm level to 2, with more attention required. Patients in Alarm level 2 should be actively evaluated for vasospasm and/or tissue damage. For example, early evidence of water accumulation in the cerebral tissue by diffusion-weighted magnetic resonance imaging would indicate inadequate oxygen delivery and necessitate corrective treatment.

More critical action is required when patients are placed in Alarm stages 3 or 4. At Alarm 3, patients should be aggressively treated. Treatment targets might include the following:

- restoration of adequate oxygen delivery (e.g., arterial hypertension)

- correction of vasospasm, if identified and amenable to endovascular treatment.

The most critical situation (Alarm 4) develops when vasospasm is refractory to treatment and tissue damage progresses. More aggressive therapies for reducing metabolic needs (e.g., barbiturates and hypothermia) or for allowing space to accommodate a swelling brain (i.e., surgical cranial decompression) could be considered at this stage. 


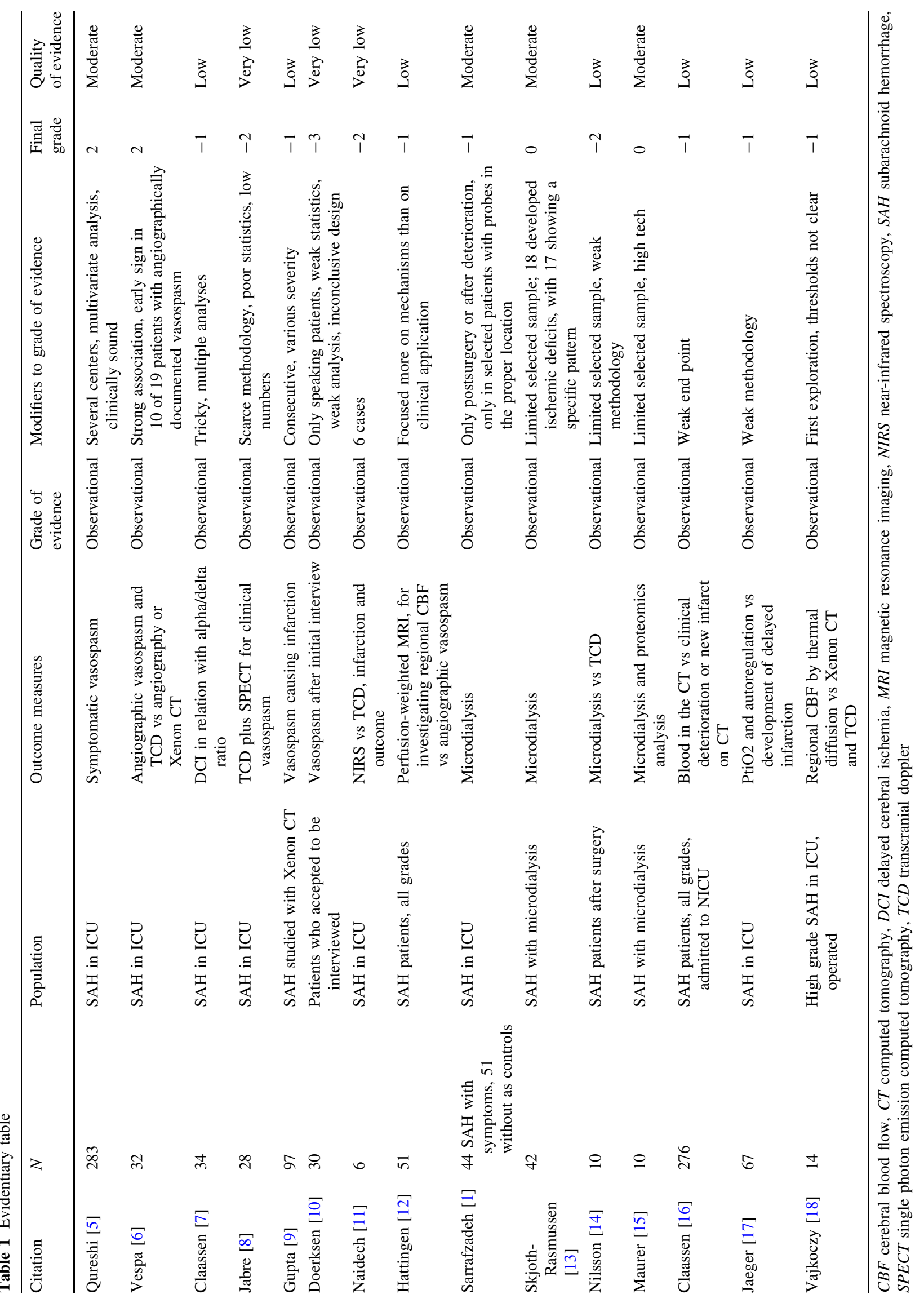


Table 2 Potential triggers for ischemia after SAH

Clinical signs:

1) Development of a new focal deficit, unexplained by other concurrent causes (e.g., hydrocephalus or re-bleeding)

2) Unexplained increases in mean arterial pressure

Monitoring data:

3) TCD worsening

4) Neuroimaging confirmation of ischemic damage (e.g., with CT, MRI, PET or other techniques)

5) Angiographic worsening of vasospasm

6) EEG signs of new ischemic lesion ${ }^{\mathrm{a}}$

7) Focal signs of inadequate oxygen delivery (e.g., by brain tissue $\mathrm{O}_{2}$ monitoring microdialysis, TCD or other newer techniques of local CBF monitoring ${ }^{\mathrm{a}}$ )

$C B F$ cerebral blood flow, $C T$ computed tomography, EEG electroencephalography, $M R I$ magnetic resonance imaging, PET positron emission tomography, $T C D$ transcranial Doppler

a For more information, see the chapter, "Monitoring for delayed cerebral ischemia: electroencephalography and invasive monitoring."
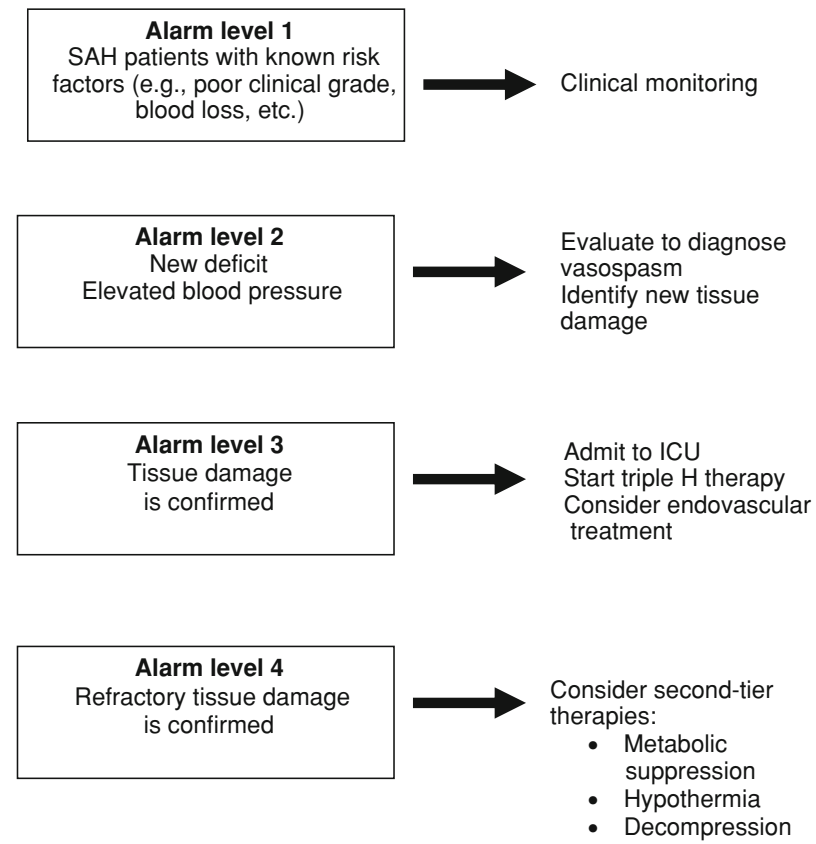

Fig. 1 Levels of alarm in SAH patients at risk for ischemic deficit. $I C U$ intensive care unit, $S A H$ subarachnoid hemorrhage; triple $\mathrm{H}$, hypervolemia, hypertension, hemodilution

\section{Conclusions}

In clinical practice, identifying clinical signs or thresholds in monitored data that predict DCI would be extremely useful. These identified triggers might then be used to reduce or prevent secondary neuronal injury after SAH. Although some data in the literature support certain factors as potential triggers, recommendations cannot be considered to be evidence based, because there are no data testing the usefulness of single triggers or their combination. Data concerning single techniques (e.g., as specificity-sensibility or associations with clinical severity or outcome) have been published, but not in this context.

Despite the absence of strong published evidence, a clinically sound approach is essential, based on physiopathology and clinical experience. Using available data does permit the development of a framework for alarm levels to guide the clinician for when additional testing/interventions will likely be warranted.

The proposed sequence of interventions, as well as the choice of triggers, should be adopted cautiously, as it is predominantly based on expert opinion rather than clinical trial data. Opinions are rated as the lowest form of evidence [22] and are, by definition, of questionable value. Moreover, the sequence of diagnosis and interventions is based on the association of vasospasm with ischemia, knowing that vasospasm is among the most frequent causes of ischemic damage; however, vasospasm is only one factor that may contribute to ischemia after SAH and other potentially important factors have not been considered here. For this reason, the diagnosis of vasospasm must be distinct from the diagnosis of inadequate cerebral delivery of oxygen and substrates.

Despite these limitations, developing and utilizing a structured sequence of interventions for capturing and counteracting ischemia after SAH is likely to be useful in clinical practice. This sequence may be used as a tool in conjunction with sound clinical practice and judgment.

\section{References}

1. Sarrafzadeh A, Haux D, Sakowitz O, et al. Acute focal neurological deficits in aneurysmal subarachnoid hemorrhage: relation of clinical course, CT findings, and metabolite abnormalities monitored with bedside microdialysis. Stroke. 2003;34:1382-8.

2. Hutter BO, Kreitschmann-Andermahr I, Gilsbach JM. Healthrelated quality of life after aneurysmal subarachnoid hemorrhage: 
impacts of bleeding severity, computerized tomography findings, surgery, vasospasm, and neurological grade. J Neurosurg. 2001;94: 241-51.

3. Al-Khindi T, Macdonald RL, Schweizer TA. Cognitive and functional outcome after aneurysmal subarachnoid hemorrhage. Stroke. 2010;41:e519-36.

4. Egge A, Waterloo K, Sjoholm H, Solberg T, Ingebrigtsen T, Romner B. Prophylactic hyperdynamic postoperative fluid therapy after aneurysmal subarachnoid hemorrhage: a clinical, prospective, randomized, controlled study. Neurosurgery. 2001;49:593-606.

5. Qureshi AI, Sung GY, Razumovsky AY, Lane K, Straw RN, Ulatowski JA. Early identification of patients at risk for symptomatic vasospasm after aneurysmal subarachnoid hemorrhage. Crit Care Med. 2000;28:984-90.

6. Vespa PM, Nuwer MR, Juhasz C, et al. Early detection of vasospasm after acute subarachnoid hemorrhage using continuous EEG ICU monitoring. Electroencephalogr Clin Neurophysiol. 1997; 103:607-15.

7. Claassen J, Hirsch LJ, Kreiter KT, et al. Quantitative continuous EEG for detecting delayed cerebral ischemia in patients with poorgrade subarachnoid hemorrhage. Clin Neurophysiol. 2004;115: 2699-710.

8. Jabre A, Babikian V, Powsner RA, Spatz EL. Role of single photon emission computed tomography and transcranial Doppler ultrasonography in clinical vasospasm. J Clin Neurosci. 2002;9: 400-3.

9. Gupta R, Crago EA, Gallek M, et al. Reduced ipsilateral hemispheric cerebral blood flow at admission is predictive of vasospasm with infarction after aneurysmal subarachnoid hemorrhage. Neurocrit Care. 2008;9:27-30.

10. Doerksen K, Naimark BJ, Tate RB. Comparison of a standard neurological tool with a stroke scale for detecting symptomatic cerebral vasospasm. J Neurosci Nurs. 2002;34:320-5.

11. Naidech AM, Bendok BR, Ault ML, Bleck TP. Monitoring with the Somanetics INVOS 5100C after aneurysmal subarachnoid hemorrhage. Neurocrit Care. 2008;9:326-31.

12. Hattingen E, Blasel S, Dettmann E, et al. Perfusion-weighted MRI to evaluate cerebral autoregulation in aneurysmal subarachnoid haemorrhage. Neuroradiology. 2008;50:929-38.

13. Skjoth-Rasmussen J, Schulz M, Kristensen SR, Bjerre P. Delayed neurological deficits detected by an ischemic pattern in the extracellular cerebral metabolites in patients with aneurysmal subarachnoid hemorrhage. J Neurosurg. 2004;100:8-15.

14. Nilsson OG, Brandt L, Ungerstedt U, Saveland H. Bedside detection of brain ischemia using intracerebral microdialysis: subarachnoid hemorrhage and delayed ischemic deterioration. Neurosurgery. 1999;45:1176-85.

15. Maurer MH, Haux D, Sakowitz OW, Unterberg AW, Kuschinsky W. Identification of early markers for symptomatic vasospasm in human cerebral microdialysate after subarachnoid hemorrhage: Preliminary results of a proteome-wide screening. J Cereb Blood Flow Metab. 2007;27:1675-83.

16. Claassen J, Bernardini GL, Kreiter K, et al. Effect of cisternal and ventricular blood on risk of delayed cerebral ischemia after subarachnoid hemorrhage: the Fisher scale revisited. Stroke. 2001;32:2012-20.

17. Jaeger M, Schuhmann MU, Soehle M, Nagel C, Meixensberger J. Continuous monitoring of cerebrovascular autoregulation after subarachnoid hemorrhage by brain tissue oxygen pressure reactivity and its relation to delayed cerebral infarction. Stroke. 2007;38:981-6.

18. Vajkoczy P, Horn P, Thome C, Munch E, Schmiedek P. Regional cerebral blood flow monitoring in the diagnosis of delayed ischemia following aneurysmal subarachnoid hemorrhage. J Neurosurg. 2003;98:1227-34.

19. Bederson JB, Connolly ES Jr, Batjer HH, et al. Guidelines for the management of aneurysmal subarachnoid hemorrhage. A statement for healthcare professionals from a special writing group of the Stroke Council, American Heart Association. Stroke. 2009;40: 994-1025.

20. Leng LZ, Fink ME, Iadecola C. Spreading depolarization: a possible new culprit in the delayed cerebral ischemia of subarachnoid hemorrhage. Arch Neurol. 2011;68:31-6.

21. Lauritzen M, Dreier JP, Fabricius M, Hartings JA, Graf R, Strong AJ. Clinical relevance of cortical spreading depression in neurological disorders: migraine, malignant stroke, subarachnoid and intracranial hemorrhage, and traumatic brain injury. J Cereb Blood Flow Metab. 2011;31:17-35.

22. Atkins D, Best D, Briss PA, et al. Grading quality of evidence and strength of recommendations. BMJ. 2004;328:1490. 